\title{
Time-delay Alignment Method of Distributed Phased Array Satellite Terminal Antenna Based on Geometric Model
}

\author{
Jing WANG \\ Institute of Advanced Communications, EECS \\ Peking University \\ Beijing, China \\ e-mail: wangjing512@pku.edu.cn \\ Jian LI \\ Institute of Advanced Communications, EECS \\ Peking University \\ Beijing, China
}

\author{
Chunyang MA \\ Institute of Advanced Communications, EECS \\ Peking University \\ Beijing, China
}

\author{
Yuxin CHENG \\ Institute of Advanced Communications, EECS \\ Peking University \\ Beijing, China
}

\author{
Jianjun WU \\ Institute of Advanced Communications, EECS \\ Peking University \\ Beijing, China \\ e-mail: just@pku.edu.cn
}

\begin{abstract}
Satellite terminal distributed phased array antenna system has a lot of advantages such as good reliability and flexibility. The delay of a signal when it arrives at the receiving end is not the same. Thus in order to combine the signals effectively, it is necessary to align the multiplexed signals in time. In this paper we propose time-delay alignment method of distributed phased array satellite terminal antenna, aiming at satellite terminals distributed phased array antenna system characteristics. Simulation results show that this method can effectively align the multiplexed signals in time and reduce complexity. The simplified scheme with dynamic strategy can also reduce the systematic error.
\end{abstract}

Keywords-distributed phased array antenna; time-delay alignment; Dynamic adjustment

\section{INTRODUCTION}

In distributed phased array antenna system, it is necessary to superimpose the received multiplexed antenna signals on the receiver. Due to different time-delay, we need to align multiple signals in time.

The delay estimation algorithm can be classified as two-way time-delay difference estimation scheme and multi-channel time-delay difference estimation scheme according to the number of signal paths to be estimated[1]. For two-way time-delay difference estimation scheme, the most classical one is the method of time-delay difference estimation based on cross-correlation. This method estimates the time-delay by searching the correlation peak between the local reference signal with the received signal[2]. LMS-based adaptive time-delay difference estimation algorithm transforms time-delay problem into the parameter estimation problem of the filter, and the minimum mean square error of the two signals is used as the criterion for the time-delay estimated[3].

For multi-antenna signals, classical methods are eigenvalue decomposition algorithm, multi-channel cross correlation algorithm and blind channel identification algorithm[4]. Eigenvalues decomposition algorithm constructs the correlation matrix of the signal by using multiple received signals with different time-delay and they are independent of each other. The time differences between the signals can be estimated according to the orthogonality between the signal and the noise. Multi-channel cross correlation algorithm generalizes the generalized cross-correlation method to the multi-channel signal. For blind channel identification algorithm, channel is equivalent to a finite impulse response filter for the time-varying linear system. The typical method is adaptive eigenvalue decomposition algorithm[5]. Besides, maximum likelihood estimation method, alternating projection method, nonlinear least squares method, highorder statistics method have been proposed.[6]

This paper mainly establishes a distributed antenna delay alignment scheme based on synthetic signal and introduces a dynamic adjustment strategy to compensate the delay difference in simplified scheme. While the dynamic adjustment strategy can also control the error within a small range.

\section{SYSTEM MODEL}

Distributed antenna technology is the utilization of antenna arrays distributed in the terminal to compose multiple signals on the joint transmission and reception, and 
synthesise the received signals effectively to improve the quality of the signals. First we should establish signal processing model of the distributed phased array antenna.

Satellite terminal distributed phased array antenna signal transmission diagram is shown in Fig. 1. It is possible to estimate the time-delay with different arrival times of signals.

$$
\tau_{i} \approx\left(\mathrm{D}_{i} \cos \theta\right) / \mathrm{v}
$$

The signal model of the satellite terminal distributed phased array antenna is as follows.

$$
x_{i}(\mathrm{t})=\alpha_{i} s\left(\mathrm{t}-\tau_{i}\right) \mathrm{e}^{j 2 \pi f(\mathrm{t}-\tau i)+\mathrm{j} \theta \mathrm{i}}+n_{i}(\mathrm{t})
$$

Thus we can consider the weighted synthesis of multiple signals as reference. The scheme can be referred to as a multi-antenna adaptive time difference estimation scheme based on synthetic signals. The schematic diagram is shown in Fig. 2.

The output signal and the synthesized signal of i-th signal after the LMS algorithm compensates for the time-delay difference are denoted respectively as $y_{i}(t)$ and $y_{c}(t)$, where $h_{i}(k)$ represents the coefficient of the filter. The error signal of i-th signal and the composite signal is as follows.

$$
e_{i}(\mathrm{t})=y_{c}(\mathrm{t})-y_{i}(\mathrm{t})=\frac{1}{N} \sum_{j=1, j \neq i}^{N} y_{i}(\mathrm{t})-\frac{N-1}{N} y_{i}(\mathrm{t})(3)
$$

The mean square error in the LMS algorithm is replaced by the instantaneous variance. Finally, the iterative updating formula of $\widehat{D_{i}}(k)$ is given as follows, where $f_{i}^{T}(k)$ is the partial derivative of $h_{i}(k)$ with respect to $\widehat{D_{i}}(k)$.

$$
\widehat{D}(\mathrm{k}+1)=\widehat{D}(\mathrm{k})-2 \frac{N-1}{N} e_{i}(\mathrm{t}) f_{i}^{T}(\mathrm{k}) \mathrm{x}_{i}(\mathrm{k})
$$

This time-delay estimation method needs to continuously receive the received signals into the delay estimation module for accurate delay alignment, which will bring a large expenditure for the system. One simplified scheme utilizes the historical estimated time-delay differences as the basis of signal delay adjustment. The scheme has greater error and result in decreased system performance while the terminal moves faster. Aiming at high-speed mobile terminal, it is necessary to take time-delay difference dynamic adjustment strategy to compensate for the time-delay difference.

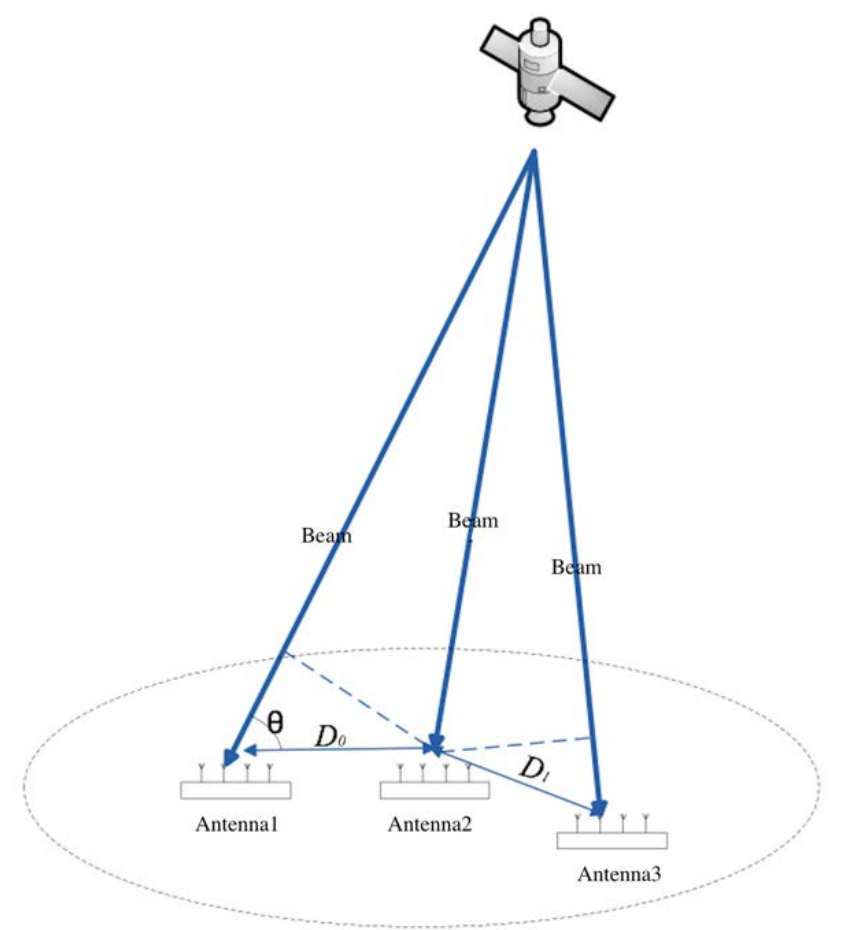

Figure 1. Signal delay difference diagram

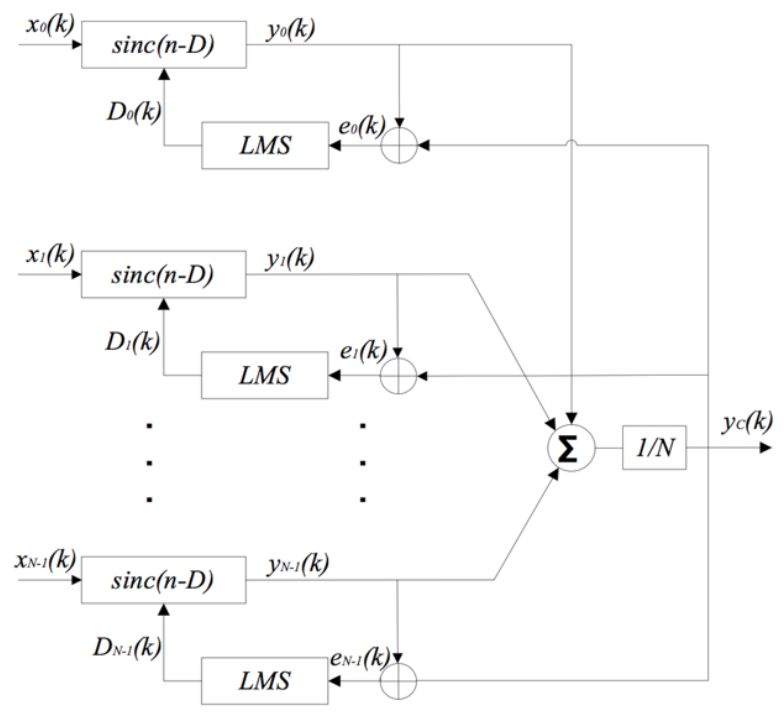

Figure 2. Schematic diagram of multi-antenna adaptive estimation scheme based on synthetic signals

\section{Time-Delay AlignMENT Method of}

Distribution Phased ARRAY SATELLite TERMinAL ANTENNA BASED ON GEOMETRIC MODEL

Through above analysis we know for achieving signal alignment, it is necessary to calculate the variation of the distance between the antennas at the time of transmission or reception and the time $\boldsymbol{\Delta} \boldsymbol{t}$ of the last time delay estimation. Fig. 3 shows the geometric structure of the variation of the path differences between the distributed antennas. 


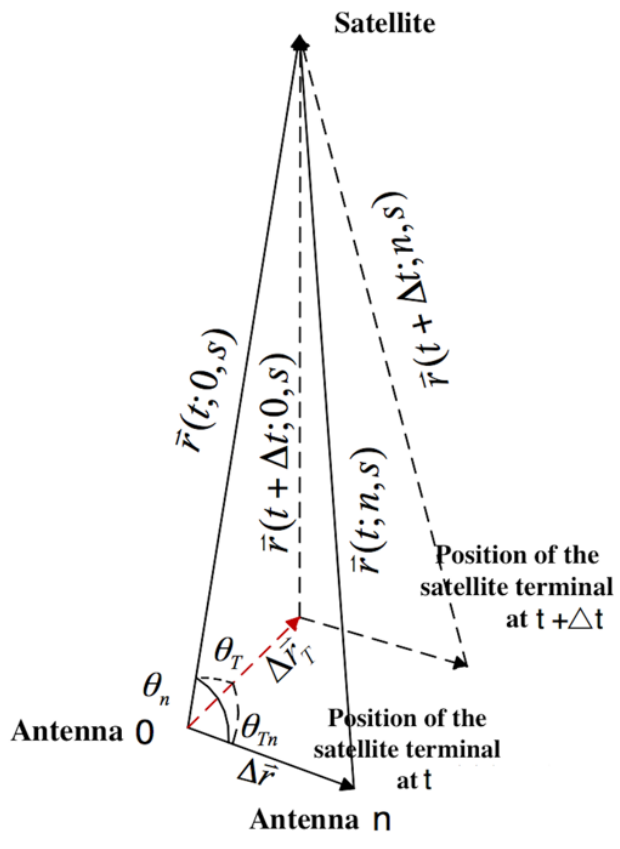

Figure 3. Schematic diagram of geometric relationship of the moving process of the satellite terminal

\section{A. Antenna Position Relationship in Geometric Model}

The vectors in the figure are defined as follows. At time $t$ the vector of the 0 -th antenna to the satellite is $r(t ; 0, s)$ and at time $t+\Delta t$ the vector is $r(t+\Delta t ; 0, s)$. So it is for the n-th antenna.

By utilizing the geometric relationship in the figure and vector expression above, we can get the positional relationship between the satellite and the different antennas at different times. At time $t$, the distance between satellite and 0 -th antenna is $d_{0}(t)=\|r(t ; 0, s)\|$ and between satellite and n-th antenna is $d_{n}(t)=\|r(t ; n, s)\|$. At time , the distance between satellite and 0 -th antenna is $d_{0}(t+\Delta t)=\|r(t+\Delta t ; 0, s)\|$ and between satellite and n-th antenna is $d_{n}(t+\Delta t)=\|r(t+\Delta t ; n, s)\|$.

\section{B. Calculate Variation of Distance Between Antennas}

At time $t$ we analyze approximate expression of the distance between n-th antenna and satellite terminal. Get the equation by using Taylor expansion and keeping the first order small quantity.

$$
\begin{aligned}
\mathrm{d}_{n}(\mathrm{t}) & =\|r(\mathrm{t} ; \mathrm{n}, \mathrm{s})\|=\left\|r(\mathrm{t} ; 0, \mathrm{~s})-\Delta r_{n}\right\| \\
& =\sqrt{r(\mathrm{t} ; 0, \mathrm{~s})^{2}+\left\|\Delta r_{n}^{2}\right\|-2\left(r(\mathrm{t} ; 0, \mathrm{~s}) \cdot \Delta r_{n}\right)} \\
& \approx\|r(\mathrm{t} ; 0, \mathrm{~s})\|-\frac{r(\mathrm{t} ; 0, \mathrm{~s}) \cdot \Delta r_{n}}{\|r(\mathrm{t} ; 0, \mathrm{~s})\|}
\end{aligned}
$$

Finally, according to the equation (5), we can get the variation of the distance difference between different earth stations and satellites in different positions.

$$
\begin{aligned}
\Delta \mathrm{d}_{n}(\mathrm{t}, \Delta \mathrm{t}) & =\left[\mathrm{d}_{n}(\mathrm{t}+\Delta \mathrm{t})-\mathrm{d}_{n}(\mathrm{t})\right]-\left[\mathrm{d}_{0}(\mathrm{t}+\Delta \mathrm{t})-\mathrm{d}_{0}(\mathrm{t})\right] \\
& \approx \frac{\Delta r_{n} \cdot \Delta r_{T}}{\|r(\mathrm{t}+\Delta \mathrm{t} ; 0, \mathrm{~s})\|}+\frac{\left[r(\mathrm{t} ; 0, \mathrm{~s}) \cdot \Delta r_{T}\right] \cdot\left[r(\mathrm{t} ; 0, \mathrm{~s}) \cdot \Delta r_{n}\right]}{\|r(\mathrm{t}+\Delta \mathrm{t} ; 0, \mathrm{~s})\| \cdot\|r(\mathrm{t} ; 0, \mathrm{~s})\|^{2}} \\
& =\frac{\Delta r_{n} \cdot \Delta r_{T}}{\|r(\mathrm{t}+\Delta \mathrm{t} ; 0, \mathrm{~s})\|}\left[-\cos \theta_{T n}+\cos \theta_{T} \cos \theta_{n}\right] \\
& \approx \frac{\Delta r_{n} \cdot \Delta r_{T}}{\mathrm{~d}_{0}(\mathrm{t})}\left[\cos \theta_{T} \cos \theta_{n}-\cos \theta_{T n}\right]
\end{aligned}
$$

It can be seen the variation of the antenna distance difference is proportional to the distance between distributed antennas $\Delta_{n}$ and also proportional to the moving distance of the satellite terminal $\Delta r_{T}$. And it is inversely proportional to the distance from the satellite to 0-th antenna.The time-delay difference of sending time which should adjust compared to time-delay estimation time is as follows.

$$
\Delta \tau=\Delta \mathrm{d} / c=\frac{\Delta r_{n} \cdot \Delta r_{T}}{\mathrm{~d}_{0}(\mathrm{t}) \mathrm{c}}\left[\cos \theta_{T} \cos \theta_{n}-\cos \theta_{T n}\right]
$$

\section{SIMULATION ANALYSIS}

Assuming there are 10 distributed phased-array antennas. The noise of each signal is zero mean Gaussian white noise and is independent. The iteration step $\mu$ is 0.05 and signal delay is $\left[-5 T_{s}, 5 T_{S}\right]$. The simulation results of two-way adaptive time delay estimation scheme and multi-channel time delay estimation scheme based on the synthesized signal are shown in Fig. 4.

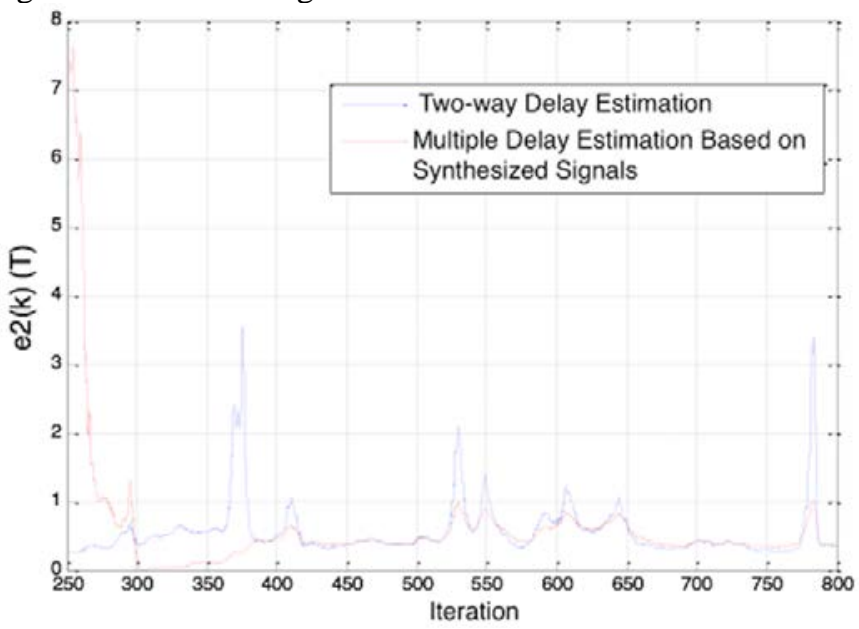

Figure 4. Delay estimation error

From the simulation results it can be seen that the time delay estimation error performance of multi-channel adaptive time-delay estimation algorithm based on the synthesized 
signal is better than two-way adaptive time delay estimation algorithm, which is consistent with the theoretical analysis of the scheme. It is shown that the scheme can improve the delay estimation performance.

The performance of scheme based on the single reference signal (scheme 1) and time delay estimation scheme based on synthesized signal as the reference signal (scheme 2) is simulated and contrasted. Set a total of 10 antennas and their signal to noise ratio is $E_{\mathrm{b}} / \mathrm{N}_{0}+[10 \mathrm{~dB}, 9 \mathrm{~dB}, \ldots, 1 \mathrm{~dB}]$.

Simulation results of time-delay estimation scheme are shown in Fig. 5.

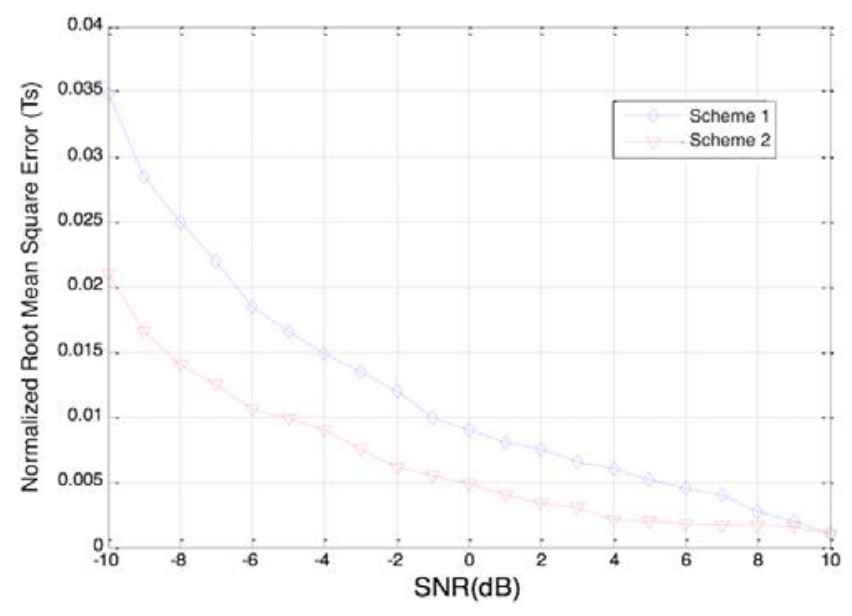

Figure 5. Comparison of the performance of time delay estimation

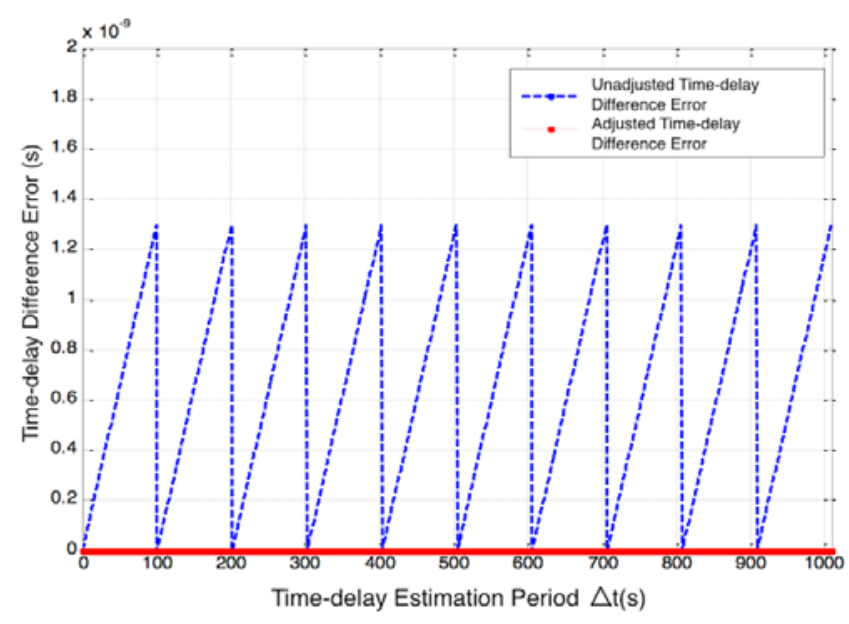

Figure 6. Time-delay error curve

From simulation result we can see the normalized mean square error of time-delay estimation based on synthesized signal is smaller than that of the two-way adaptive time-delay estimation, which bring a certain degree of performance improvement, without undue impact on the complexity.

Speed of terminal is $1000 \mathrm{~km} / \mathrm{h}$ and interval between two antennas is $50 \mathrm{~m}$. If period between two time-delay estimation is $100 \mathrm{~s}$, the time-delay error between the two signals with and without the delay adjustment strategy is shown in Fig. 6.

It can be seen time-delay differences of simplified scheme signal show a zigzag variation, because signal only estimates time-delay once in every period. This error can not be corrected until next period. If we can adjust dynamically in one time-delay estimation period, the delay difference can keep a stable level. The estimated delay difference can be utilized directly as the basis for signal alignment.

\section{SUMMARY}

This paper mainly proposes a distributed antenna delay alignment scheme based on synthetic signal and simplify it. The dynamic adjustment strategy is introduced to compensate the delay difference in the simplified scheme. Simulation results show that the proposed method can improve the performance of time delay estimation. The dynamic adjustment strategy in the simplified scheme can also control error within a small range.

\section{ACKNOWLEDGEMENTS}

This work is supported by the National Natural Science Foundation of China (No.61371073). Corresponding author: Jianjun Wu; Phone: +86-10-62752848; Email: just@pku.edu.cn.

\section{REFERENCES}

[1] C. Shen and H. Yu. Time-delay alignment technique for a randomly distributed sensor array [J]. IET Commun., 2011, 5(8): 1068-1072.

[2] Jacob Benesty, Yiteng Huang and Jingdong Chen. Time delay estimation via minimum entropy [J]. IEEE Signal Processing Letters, 2007, 14(3): 157-160.

[3] Jingdong Chen and Yiteng Huang. Robust time delay estimation exploiting redundancy among multiple microphones [J]. IEEE Transactions on Speech and Audio Processing, 2003, 11(6): 549-557.

[4] Ianniello J P. High-resolution multipath time delay estimation for broad-band random signals [J]. Acoustics, Speech and Signal Processing, IEEE Transactions on, 1988, 36(3): 320-327.

[5] Reed F, Feintuch P L, Bershad N J. Time delay estimation using the LMS adaptive filter-static behavior [J]. Acoustics, Speech and Signal Processing, IEEE Transactions on, 1981, 29(3):561-571.

[6] Zhang Yao, Ji Da-xiong, Wu Jian-guo and Gao Lei. Multipath time-delay estimate based on homomorphic filtering in logarithm domain[J]. OCEANS 2014 - TAIPEI, 2014, 1-4. 